\title{
Impact of arterial and venous cannula diameter in venoarterial extracorporeal membrane oxygenation (VA-ECMO)
}

\author{
A Supady ${ }^{1,2}$, T Wengenmayer ${ }^{1,2}$, F Schroth $^{1,2}$, D Duerschmied ${ }^{1,2}$, C Benk $^{3}$, C Bode $^{1,2}$, DL Staudacher B $^{1,2}$ \\ ${ }^{1}$ Department of Medicine III (Interdisciplinary Medical Intensive Care), Medical Center, University of Freiburg, Faculty of Medicine, University of Freiburg, Germany; \\ ${ }^{2}$ Department of Cardiology and Angiology I, University Heart Center Freiburg University, Germany \\ ${ }^{3}$ Department of Cardiovascular Surgery, University Heart Center Freiburg University, Germany
}

Background: For cannulation in venoarterial extracorporeal membrane oxygenation (VA-ECMO) therapy, cannula with different diameters are available. While blood flow through a cannula is estimated by the Hagen-Poiseuille equation and thus dependent on diameter and length, in vivo data is missing.

Methods: All patients with VA-ECMO cannulated at a tertiary referral hospital were included in a registry. Once every 24 hours within the first 7 days after cannulation, VA-ECMO blood flow and pump speed were recorded. Resistance of the ECMO circuit (blood flow divided by 1000 rounds per minute (RPM)), was calculated and pooled for this research. The Sorin Centrifugal Pump Console was used as ECMO system and HLS cannulae (Maquet) with a length of $23 \mathrm{~cm}$ for arterial and $55 \mathrm{~cm}$ for venous drainage.

Results: A total of 216 patients and 653 data points were evaluated. Average Blood flow was $3.62 \pm 1.1 \mathrm{l} / \mathrm{min}$ with pump speed of $2677 \pm 601 \mathrm{RPM}$. Most commonly used cannulas were $23 \mathrm{Fr}$. venous and $17 \mathrm{Fr}$. arterial (81 patients) followed by 23/15Fr. (66 patients), 21/17Fr. (30 patients), and 21/15Fr. (39 patients). Normalized blood flow was highest in the 23/17 Fr. group $(1,42 \mathrm{l} / \mathrm{min} / 1000 \mathrm{RIM})$ and lowest in the $23 / 15 \mathrm{Fr}$. group $(1,27 \mathrm{l} / \mathrm{min} / 1000 \mathrm{RPM})$. Concerning cannula size, there was no significant difference in blood flow in the 23Fr. and 21Fr. venous cannulas while arterial size impacted blood flow significantly with an average of $0.13 \mathrm{l} / \mathrm{min} / 1000 \mathrm{RIM}$ lower blood flow in the smaller cannulas (see figure 1).
Conclusion: Blood flow to patients with VA-ECMO is primarily limited by diameter of the arterial cannula. In larger arterial cannulas, also venous cannula diameter impacts resistance of the ECMO circuit.

Figure 1: VA-ECMO blood flow in patients cannulated with different cannula diameters and combinations.

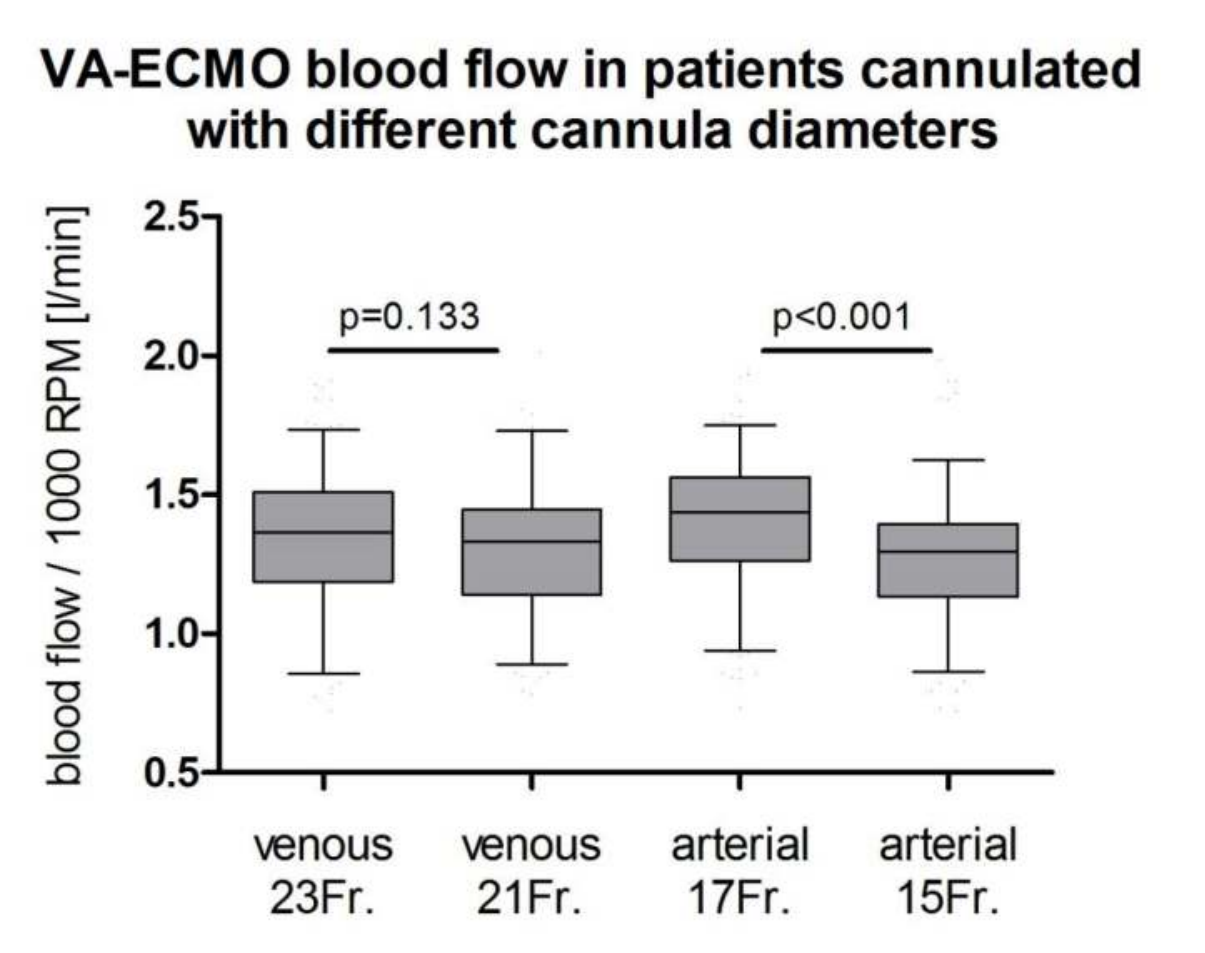

VA-ECMO blood flow in patients cannulated with different cannula combinations

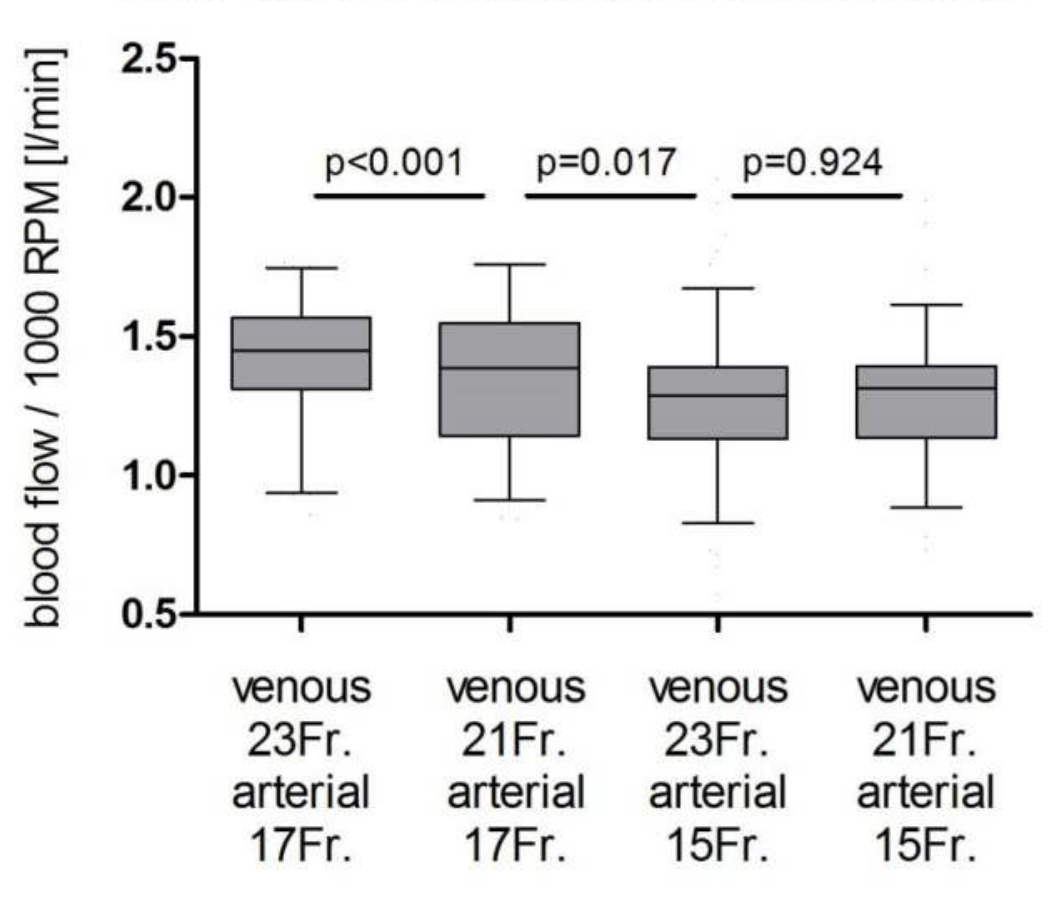

